\title{
The Influence of Pregnancy on the Recurrence of Cutaneous Malignant Melanoma in Women
}

\author{
M. Albersen, ${ }^{1}$ V. I. Westerling, ${ }^{2}$ and P. A. M. van Leeuwen ${ }^{3}$ \\ ${ }^{1}$ Department of Metabolic and Endocrine Diseases, Wilhelmina Children's Hospital, \\ University Medical Centre Utrecht, 3584 EA Utrecht, The Netherlands \\ ${ }^{2}$ Department of General Practice, University Medical Centre Utrecht, $3732 \mathrm{HJ}$ De Bilt, The Netherlands \\ ${ }^{3}$ Department of Surgical Oncology, VU University Medical Centre, 1081 HV Amsterdam, The Netherlands \\ Correspondence should be addressed to P. A. M. van Leeuwen, pam.vleeuwen@vumc.nl \\ Received 25 May 2010; Accepted 24 June 2010 \\ Academic Editor: Ashfaq A. Marghoob
}

Copyright (๑) 2010 M. Albersen et al. This is an open access article distributed under the Creative Commons Attribution License, which permits unrestricted use, distribution, and reproduction in any medium, provided the original work is properly cited.

Objective. The aim of this study was to determine whether pregnancy increases the recurrence risk of cutaneous malignant melanoma (CMM) in women with a history of stage I CMM. Methods. The electronic medical databases of Medline and Embase were explored. All 1084 obtained articles were screened on title and abstract using predetermined inclusion and exclusion criteria. A critical appraisal of relevance and validity was conducted on the remaining full text available articles. Results. Two studies were selected. Both studies revealed no significant difference in disease-free survival between women with stage I CMM and the control population. Conclusion. Pregnancy does not increase the recurrence risk of CMM in women with a history of stage I CMM.

\section{Clinical Scenario}

A 33-year-old nulliparous woman was seen by the dermatologist because of a mole on her leg, which had been growing and itching over the past year. After excision of the lesion and pathological examination, the diagnosis made was a cutaneous malignant melanoma, with Clark level IV, a Breslow thickness of $1.25 \mathrm{~cm}$-and tumor-free margins. Because excision had been successful, only a sentinel lymph node dissection was conducted which yielded no metastatic spread. The conclusive diagnosis therefore was a stage I cutaneous malignant melanoma. Every three months now, the patient is seen by the dermatologist in order to keep a close watch on the development of recurrence. Currently, she and her partner would like to embark on a first pregnancy.

\section{Introduction}

The incidence of Cutaneous Malignant Melanoma (CMM) has shown a rapid increase over the past decades, therefore substantiating a growing problem. About $27 \%$ of the noninvasive CMM diagnosed in the Netherlands in 2003 were seen in women between 30 and 44 years of age, of which $13 \%$ eventually died because of the disease. The currently known factors determining outcome are mostly the skin location of the melanoma, the Breslow thickness, and the Clark level $[1,2]$. Keeping in view the possibility of tumor sensitivity to hormones, although the possible mechanism of this influence is still unknown, the question arises whether pregnancy influences the recurrence of CMM [2]. In the past, some studies have suggested a negative influence $[3,4]$, whereas other studies showed no effect of pregnancy on the prognosis of cutaneous malignant melanoma $[5,6]$. Therefore, the aim of this article is to answer the following clinical question:

does [pregnancy] increase the [recurrence) risk (of cutaneous malignant melanoma] in [women with a history of stage I cutaneous malignant melanoma]?

Pregnancy was defined as occurring within five years of CMM diagnosis in previously nulliparous women. Stage I cutaneous melanoma was defined as a malignant melanoma localized to the site of origin on the skin, with no evidence of regional or distant spread [7]. Recurrence was defined as a subsequent manifestation of CMM within ten years of initial 


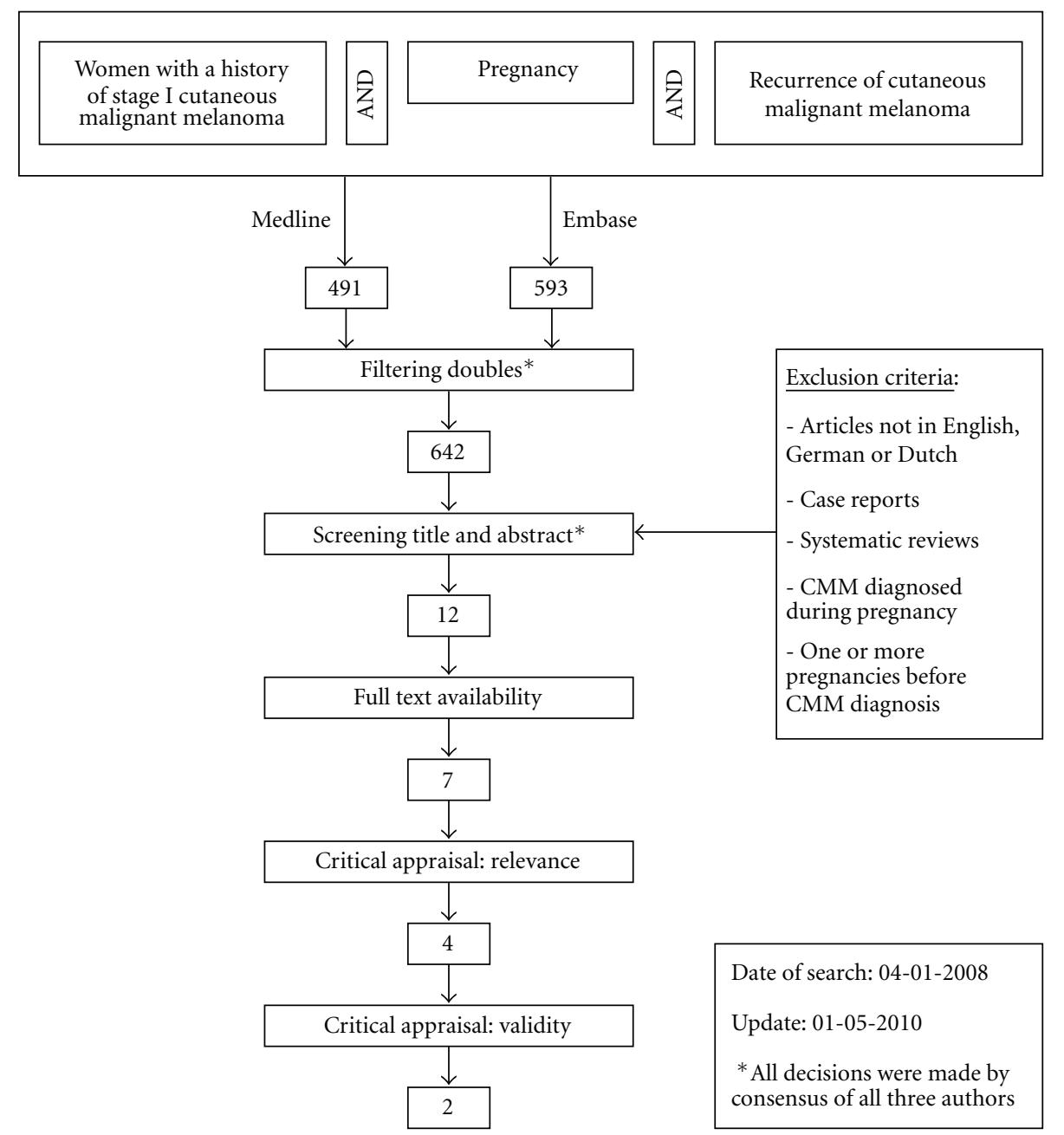

Figure 1: Flow chart.

diagnosis and treatment. In addition, the disease-free survival is a period of time in which no CMM recurrence is seen.

\section{Methods}

3.1. Search and Selection. Synonyms for domain, determinant and outcome were identified using Thesaurus, Embase EMTREE-tool, Medline MesH-terms, and Index [Title/Abstract] and were applied in the electronic databases of Embase and Medline. This full cover search resulted in 491 articles in Medline and 593 articles in Embase (Table 1). The title and abstract of a total of 1084 articles were subsequently screened for their relevance concerning the clinical question, based on the following inclusion criteria: pregnancy within five years of CMM diagnosis in previously nulliparous women, the primary diagnosis being stage I cutaneous malignant melanoma, and CMM recurrence within ten years of the primary diagnosis. The exclusion criteria are shown in the flow chart (Figure 1). All decisions were made through a consensus of all three authors. Upon screening, 12 articles remained for further analysis. A total of seven articles appeared to be full text available.
TABLE 1: Search strategy.

\begin{tabular}{llc}
\hline Database & Search $^{\mathrm{a}}$ & Hits \\
\hline & ((Woman OR Women OR Female OR & \\
& Females OR Patient OR Patients) AND & \\
& (Skin OR Cutaneous OR Malignant OR & \\
& Melanoma OR Melanomas)) AND & \\
& (Pregnancy OR Pregnancies OR Pregnant & \\
Medline & OR Gravidity OR Gravidities OR & 491 \\
& Gestation) AND (Recurrent OR & \\
& Recurrence OR Recurrences OR & \\
& Recurring OR Recidive OR Recidives OR & \\
& Recidivism OR Recidivisms OR & \\
& Recidivating OR Recidivation OR & \\
& Relapsing OR Relapse OR Relapses) & \\
\hline Embase & Idem Medline & \\
\hline
\end{tabular}

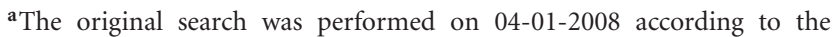
strategy above.

An update performed on 01-05-2010 following the same search strategy yielded no additional relevant articles.

3.2. Critical Appraisal. The selected articles were screened for relevance of domain, determinant, and outcome. As a 


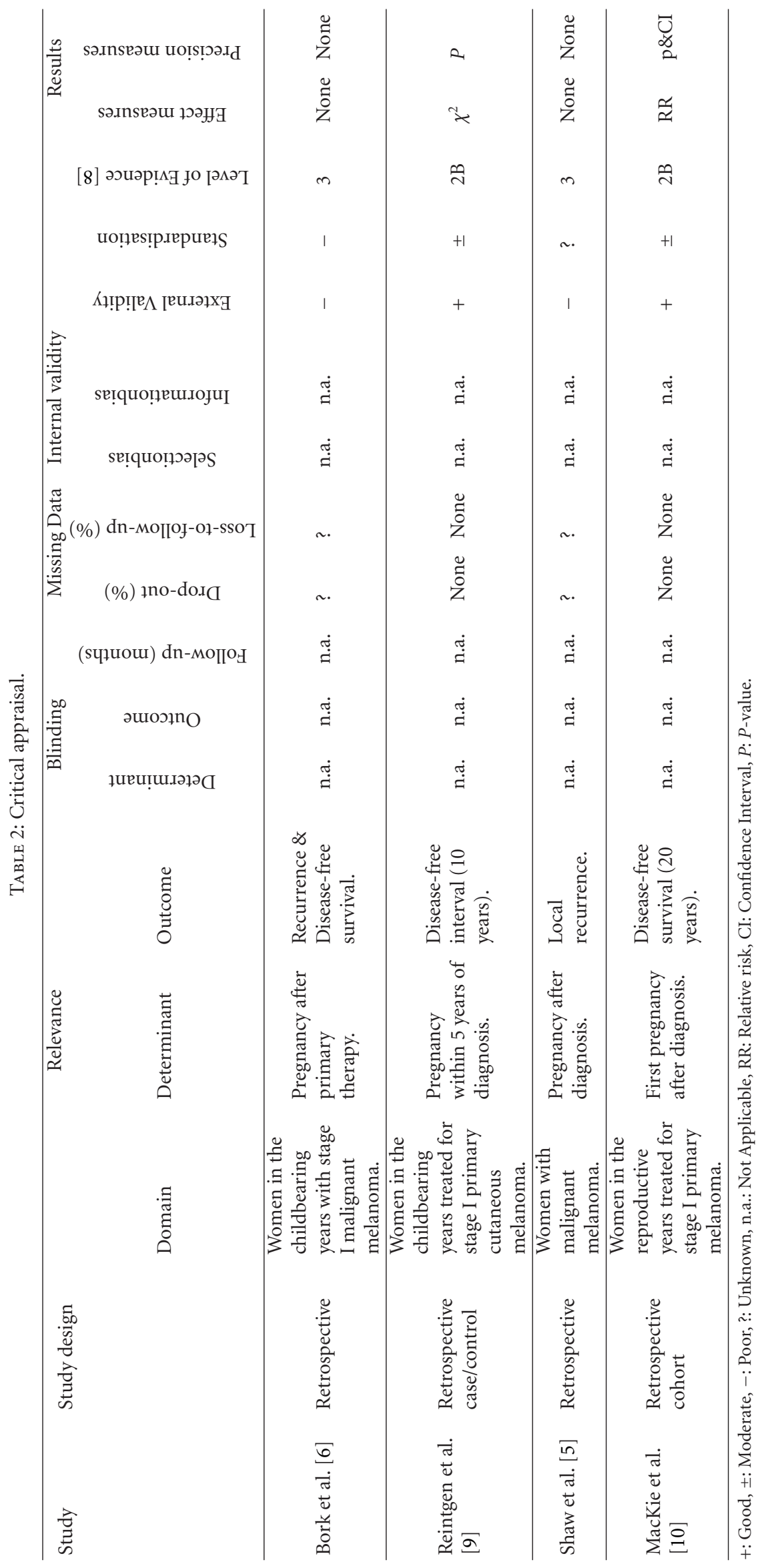


TABLE 3: Influence of pregnancy on recurrence of cutaneous malignant melanoma in women.

\begin{tabular}{|c|c|c|c|c|c|}
\hline \multirow{2}{*}{ Study } & \multirow{2}{*}{ Population $(n)$} & \multicolumn{2}{|c|}{ Effect } & \multicolumn{2}{|c|}{ Precision } \\
\hline & & $\chi^{2}(*)$ & $\mathrm{RR}$ & $P$-value & $95 \% \mathrm{CI}$ \\
\hline \multirow{2}{*}{ Reintgen et al. [9] } & 43 patients & 0.04 univariate & - & .80 univariate & - \\
\hline & 585 controls & 0.0 multivariate & - & 1.00 multivariate & - \\
\hline \multirow{2}{*}{ MacKie et al. [10] } & 85 patients & - & $1.21(* *)$ & .66 & $0.52-2.79$ \\
\hline & 143 controls & - & $0.71(* *)$ & .54 & $0.23-2.15$ \\
\hline
\end{tabular}

$\left({ }^{*}\right)$ With $\chi^{2}$, the chi-square test is pronounced, which was used to test the significance of differences between the patient and the control groups. A concomitant $P$-value $>.05$ means that a correlation between pregnancy and CMM recurrence is not likely, and that if there is a correlation at all, this is not very strong, as suggested by the small $\chi^{2}$-values of 0.04 and 0.0 in the univariate and multivariate analyses, respectively.

$(* *)$ Compared to women diagnosed with a CMM in between pregnancies.

result, four articles remained for validity screening. These articles were evaluated on the basis of the components shown in Table 2. Because Shaw [5] and Bork et al. [6] failed to report any effect or precision measures and did not compare patients with controls, these studies were excluded from further assessment. In contrast, Reintgen et al. [9] and MacKie et al. [10] did meet the criteria for a valid study with a level of evidence (Offringa et al. [8]) of 2B and were therefore considered valuable for answering the clinical question.

\section{Results}

Reintgen et al. [9] set up a retrospective case control study, whose objective was to assess whether pregnancy within five years of diagnosis would influence the ten-year diseasefree interval in women with stage I cutaneous malignant melanoma. Patients were matched with a control population consisting of female stage I CMM patients between the age of 15 and 44 years, who were not pregnant either at diagnosis or within five-years of diagnosis. The study showed that the ten-yeardisease-free interval of the patient group did not significantly differ from that of the control group (Table 3 ).

MacKie et al. [10] also established a retrospective case control study, in which the effect of pregnancy on the twenty year-disease-free survival after diagnosis of stage I cutaneous malignant melanoma was investigated. Patients were matched with a control population of women who had completed all pregnancies before a stage I CMM was diagnosed. The study yielded a relative risk of 1.21, which would suggest a slight effect of pregnancy on CMM recurrence. After regression analysis however, this did not prove to be significant (Table 3 ).

\section{Conclusion}

According to both Reintgen et al. [9] and MacKie et al. [10], pregnancy does not increase the recurrence risk of cutaneous malignant melanoma in women with a history of stage I cutaneous malignant melanoma.

\section{Discussion}

This evidence-based case report suggests that there is no negative influence of pregnancy on the recurrence of cuta- neous malignant melanoma in women. There are however a number of drawbacks with respect to the critically appraised studies. Firstly, both Reintgen et al. [9] and MacKie et al. [10] used only a small number of patients compared to the relatively large control groups that were included. Secondly, in the study of MacKie et al. [10] the results of the patient group were not compared with the results of the control group, but both groups were compared with women diagnosed with a CMM in between pregnancies. Reintgen et al. [9] on the other hand used a more realistic control group composed of nulliparous women with a CMM diagnosis. Worth mentioning is the use of a regression analysis in both the study of Reintgen et al. [9] and that of MacKie et al. [10], which is a reliable statistical analysis method to determine the effect of possible confounders.

\section{Recommendation}

The best available evidence does not show any effect of pregnancy on the recurrence risk of cutaneous malignant melanoma in women. Nevertheless, a careful recommendation should be given, with Breslow thickness, Clark level, and skin localization taken into account. Because recurrence, independent of pregnancy, is most likely to develop within two to three years after CMM diagnosis and treatment $[11,12]$, and since both diagnostic and therapeutical interventions are possibly harmful to the unborn child, appropriate timing is necessary. Women should therefore consider delaying pregnancy for at least two to three years, taking into account the concomitant risk of maternal age related problems such as infertility and fetal developmental abnormalities.

\section{Acknowledgment}

MA and VIW contributed equally to this paper.

\section{References}

[1] Netherlands Cancer Registry, "Incidence of Cancer in the Netherlands".

[2] M. S. Driscoll and J. M. Grant-Kels, "Hormones, nevi, and melanoma: an approach to the patient," Journal of the American Academy of Dermatology, vol. 57, no. 6, pp. 919-931, 2007. 
[3] G. T. Pack and I. M. Scharnagel, "The Prognosis for Malignant Melanoma in the Pregnant Woman," Cancer, vol. 4, pp. 324-334, 1951.

[4] B. F. Byrd Jr. and W. J. McGanity, "The effect of pregnancy on the clinical course of malignant melanoma," Southern medical journal, vol. 47, no. 3, pp. 196-200, 1954.

[5] J. H. F. Shaw, "Malignant melanoma in Auckland, New Zealand," Surgery Gynecology and Obstetrics, vol. 166, no. 5, pp. 425-430, 1988.

[6] K. Bork, W. Brauninger, and B. M. Czarnetzki, "Schwangerschaft nach Malignem Melanom: Verlaufsbeobachtung bei 23 Patientinnen," Deutsche Medizinische Wochenschrift, vol. 110, no. 35, pp. 1323-1327, 1985.

[7] S. Retsas and M. J. Mastrangelo, "Reflecting on the 2001 American Joint Committee on Cancer Staging System for melanoma," Seminars in Oncology, vol. 34, no. 6, pp. 491-497, 2007.

[8] M. Offringa, W. J. J. Assendelft, and R. J. P. M. Scholten, Inleiding in evidence-based medicine: klinisch handelen gebaseerd op bewijsmateriaal, Bohn Stafleu van Loghum, Houten, The Netherlands, 2nd edition, 2003.

[9] D. S. Reintgen, K. S. McCarthy Jr., and R. Vollmer, "Malignant melanoma and pregnancy," Cancer, vol. 55, no. 6, pp. 1340-1344, 1985.

[10] R. M. MacKie, R. Bufalino, A. Morabito, C. Sutherland, and N. Cascinelli, "Lack of effect of pregnancy on outcome of melanoma," Lancet, vol. 337, no. 8742, pp. 653-655, 1991.

[11] W.-J. Poo-Hwu, S. Ariyan, L. Lamb et al., "Follow-up recommendations for patients with American Joint Com-mittee on cancer stages I-III malignant melanoma," Cancer, vol. 86, no. 11, pp. 2252-2258, 1999.

[12] T. R. Fincher, T. M. McCarty, T. L. Fisher et al., "Patterns of recurrence after sentinel lymph node biopsy for cutaneous melanoma," American Journal of Surgery, vol. 186, no. 6, pp. 675-681, 2003. 


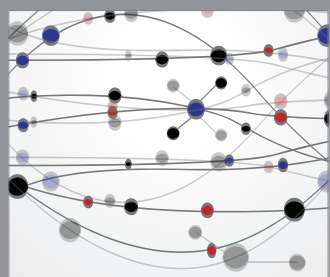

The Scientific World Journal
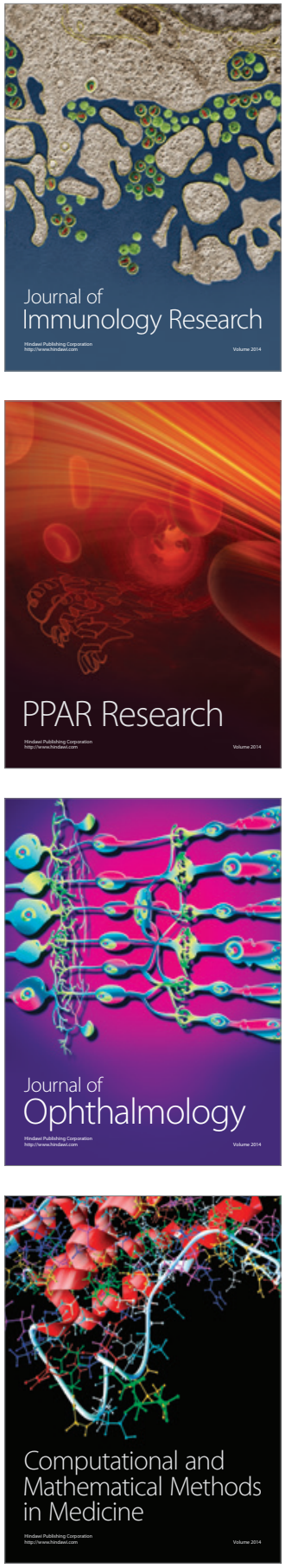

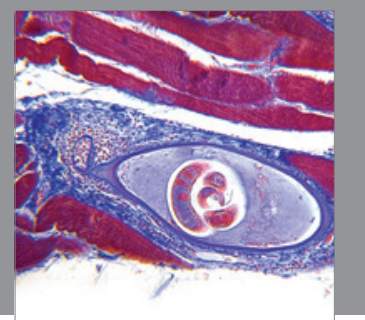

Gastroenterology

Research and Practice
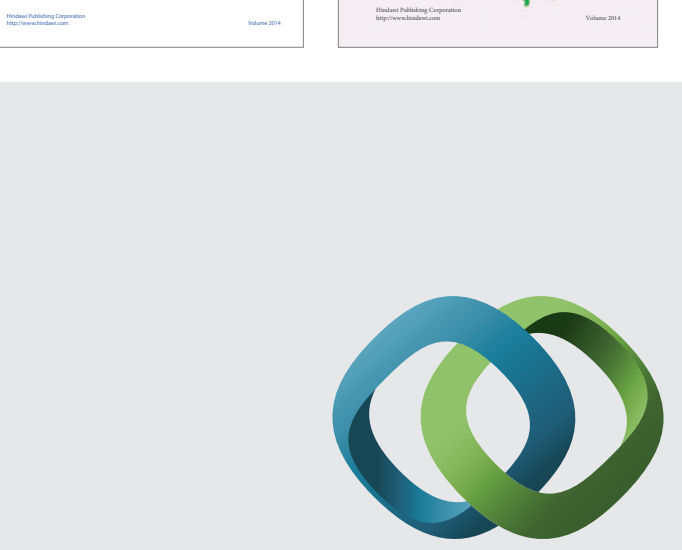

\section{Hindawi}

Submit your manuscripts at

http://www.hindawi.com
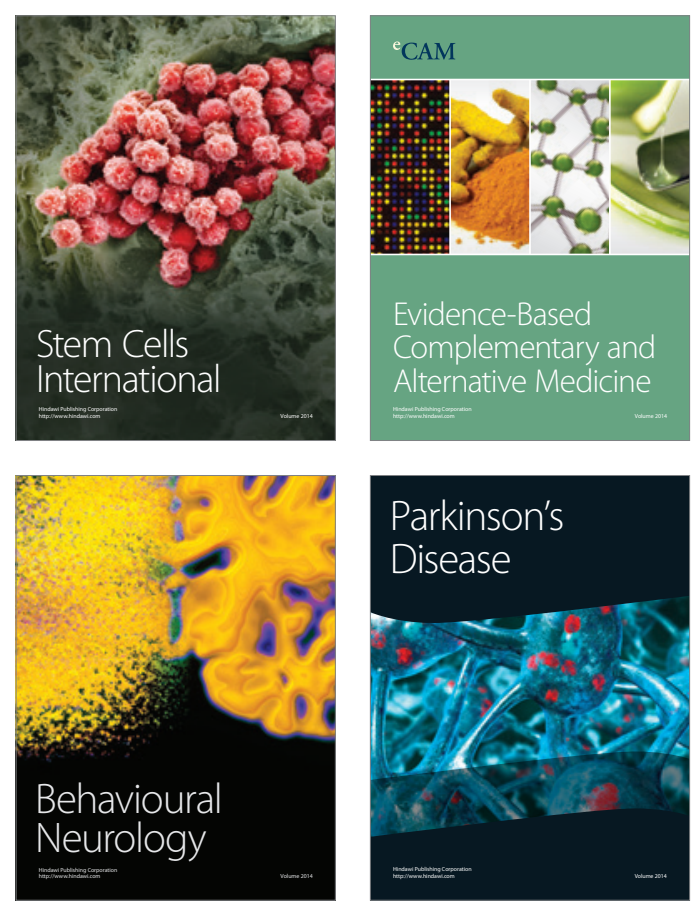

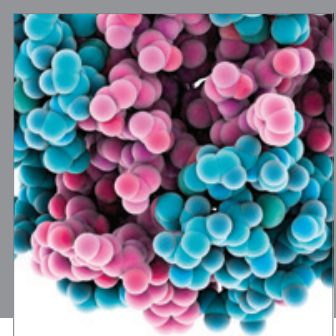

Journal of
Diabetes Research

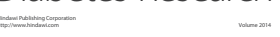

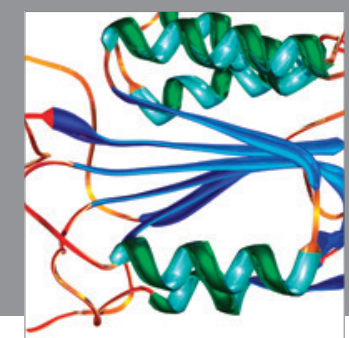

Disease Markers
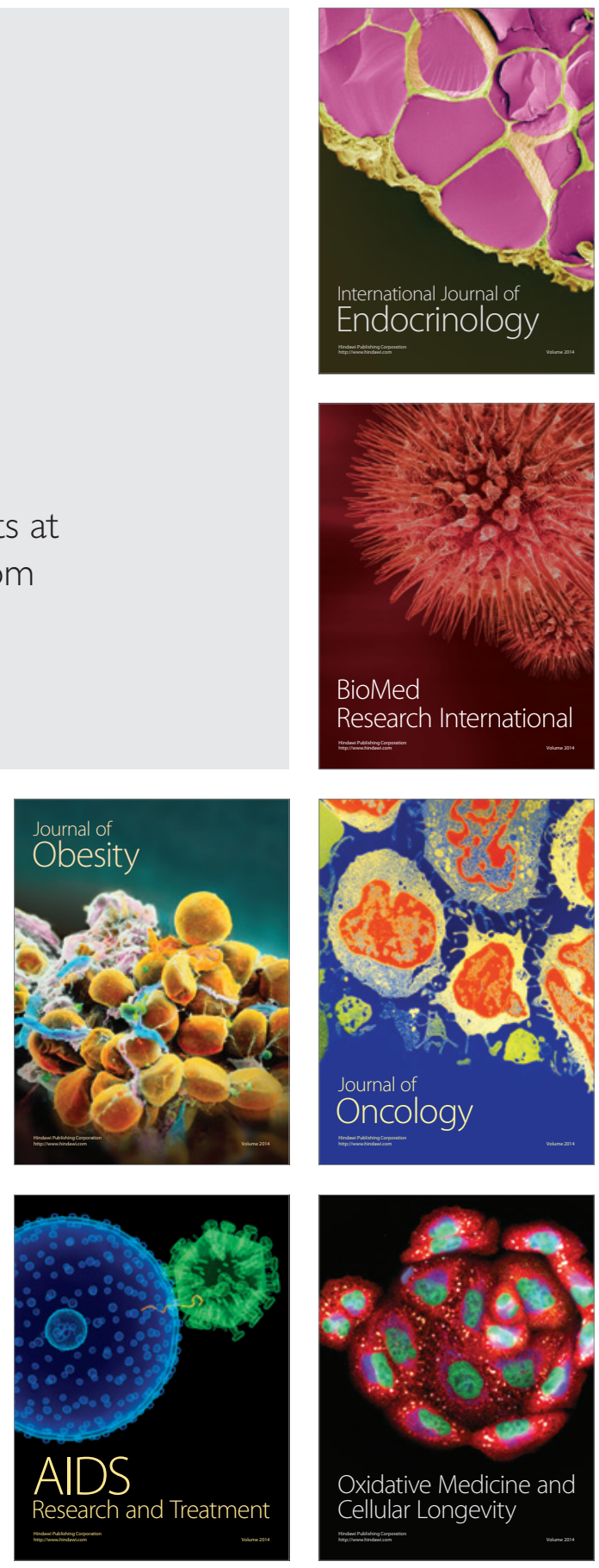